\title{
Modulation of amyloid $\beta$ protein precursor processing as a means of retarding progression of Alzheimer's disease
}

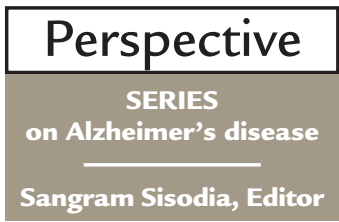

\author{
Steven L. Wagner and Benito Munoz \\ SIBIA Neurosciences Inc., La Jolla, California 92037-4641, USA
}

Address correspondence to: Steven L. Wagner, SIBIA Neurosciences Inc., La Jolla, California 92037-4641, USA. Phone: (858) 452-5892 ext. 406; Fax: (858) 452-9279; E-mail: swagner@sibia.com.

Alzheimer's disease (AD), a chronic neurodegenerative disorder, is characterized neuropathologically by an abundance of extracellular neuritic plaques (NPs) containing large amounts of a predominantly fibrillar peptide termed amyloid $\beta(A \beta)$. The brains of victims of AD also harbor excessive numbers of neurofibrillary tangles (NFTs). NFTs are intraneuronal accumulations of paired helical filaments composed primarily of hyperphosphorylated isoforms of the microtubule-associated protein tau. In the United States, a definitive diagnosis of AD is based, at autopsy, on histopathological evidence of high numbers of both NPs and NFTs in individuals having a previous clinical history of progressive dementia. Additional neuropathological changes typically accompanying $\mathrm{AD}$ include congophilic angiopathy ( $\mathrm{A} \beta$ fibrils within the basement membrane of the cerebrovasculature) and selective neuronal cell loss.

$\mathrm{AD}$ can be subdivided into early-onset $\mathrm{AD}$ (EOAD) and late-onset $\mathrm{AD}$ (LOAD), depending on whether symptoms arise before or after the age of 60 years. EOAD is inherited as an autosomal dominant disorder and can arise from mutations in at least 3 different genes (see the Perspective by Tanzi in the previous issue of the JCI). The first EOAD mutation identified was a missense mutation in the amyloid precursor protein $(A P P)$ gene, located on chromosome 21 (1). Several other missense mutations in APP have also been shown to segregate with EOAD. The second gene implicated in EOAD, localizing to chromosome 14, was designated presenilin 1 (PS1; ref. 2 ). Subsequently, a relatively large number of mutations (>50) have been reported in PS1 in patients with EOAD (3). Finally, EOAD-linked mutations have been found in a few rather large AD kindreds in presenilin 2 (PS2), which resides on chromosome 1 and exhibits extensive sequence homology with PS1 (4).

APP proteolytic processing in vitro and in vivo. The first of the many EOAD mutations (717 Val to Ile or "London") was identified in a region of $A P P$ directly adjacent to that encoding the $A \beta$ peptide (Figure 1 ). Because $A \beta$ is known to be generated through the proteolytic processing of $\mathrm{APP}(5)$, the proximity of the mutations to the A $\beta$ motif suggested that these mutations influence proteolytic processing in a manner that promotes amyloidogenesis (6). Several studies have since revealed that missense mutations near the $\mathrm{A} \beta$ domain can influence the rate and amount of $A \beta$ peptide production in vitro and in vivo. The 670/671 (Lys-Met to Asn-Leu or "Swedish") double mutation, positioned directly adjacent to the
$\mathrm{NH}_{2}$-terminus of the $\mathrm{A} \beta$ domain within APP, enhances the production of $A \beta$ by approximately 6 -fold (7). In addition, missense mutations positioned near the $\mathrm{COOH}$-terminus of the $\mathrm{A} \beta$ motif (i.e., V717I or "London" and V717F or "Indiana") appear to cause an increase in the amount of a longer $A \beta$ peptide $\left(A \beta_{42}\right)$ relative to the amount of a shorter, but normally more abundant, $A \beta$ peptide $\left(A \beta_{40}\right)$. Interestingly, the longer form, $A \beta_{42}$, is more frequently found associated with neuritic plaques (8) and is more fibrillogenic in vitro. Elegant kinetic studies have suggested that $A \beta_{42}$ may serve as a "seed" in the nucleation-dependent polymerization of ordered noncrystalline A $\beta$ peptides (9).

More recently, mutations in PS1 and PS2 have been found that appear to influence APP proteolytic processing, causing an increase in the relative amounts of the longer, more fibrillogenic $A \beta_{42}$ (10). Increased levels of $\mathrm{A} \beta_{42}$ appear to be a common biochemical feature of all of the EOAD-segregating mutations. A $\beta$ peptides (especially $A \beta_{42}$ ) have been shown to be capable of inducing programmed cell death in cultured primary neurons (11), possibly by invoking toxic levels of hydrogen peroxide to accumulate in cells (12); generating reactive oxygen species (13); stimulating nitric oxide production through an NFKB-dependent mechanism (14); binding C1q and activating the classical pathway of complement (15); and accelerating the tissue plasminogen activator (TPA)mediated activation of plasminogen to plasmin (16), to cite just a few mechanisms. From a pathophysiological point of view, $A \beta$ peptides, and especially $A \beta_{42}$, appear to play a very complex role in disease activity. The pathogenetic findings, combined with a rather voluminous body of literature, implicate $\mathrm{A} \beta$ peptides in some neurotoxic and/or pathophysiological process and strongly suggest that therapeutic approaches aimed at modulating the proteolytic catabolism of APP or reducing $A \beta_{42}$ formation might provide fruitful clinical results.

Currently, there are several screening strategies directed toward the discovery of molecules capable of modulating one or more of the detrimental properties of $A \beta$ peptides, each with the ultimate goal of identifying compounds capable of retarding the progression of $\mathrm{AD}$. These strategies include screens designed to identify antagonists of $A \beta$ neurotoxicity (17), inhibitors of the neuroglial-mediated immunoinflammatory response to $A \beta$ (18), molecules capable of preventing A $\beta$ fibrillogenesis (19), mAb's that can disaggregate $A \beta$ fibrils (20), and compounds capable of inhibiting $A \beta$ deposition onto synthetic immobilized $A \beta$ 


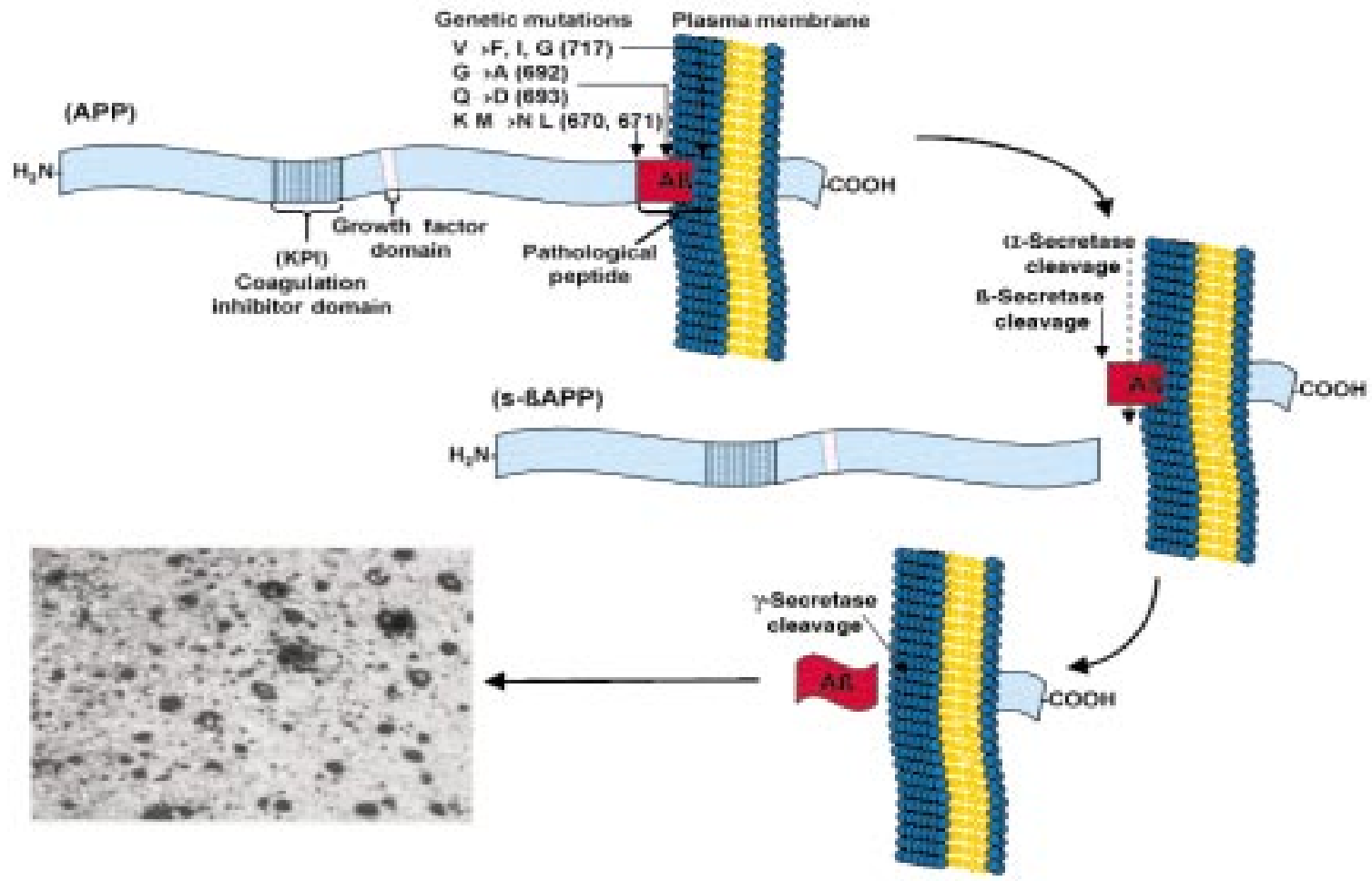

Figure 1

Schematic representation of APP proteolytic processing and the pathways leading to neuritic plaque formation in Alzheimer's disease.

fibrils (21). The approach we describe in detail here is to identify compounds able to reduce the cellular capacity for synthesizing intact $A \beta$ peptides by either direct or indirect modulation of the amyloidogenic proteolysis of APP.

Cell-based drug screening strategies. Inhibition of A $\beta$ peptide formation through either $\beta$ - or $\gamma$-secretase modulation is an attractive therapeutic approach for the treatment for AD. Although major efforts have been mounted in both academic and industrial laboratories to unequivocally identify the enzymes responsible for the proteolytic events leading to $\mathrm{A} \beta$ formation, both remain unknown entities. When a target proteinase enzyme is well characterized, the task of designing and optimizing low-molecular-weight inhibitors, while challenging, is reasonably straightforward. In contrast, when a target enzyme is either poorly characterized, or in the case of either $\beta$ - or $\gamma$-secretase, completely unknown, the rational design of inhibitors, which relies on the knowledge of the enzyme class and the ability to measure kinetic parameters, is not directly applicable. Therefore, a major contemporary therapeutic challenge is the design of compounds capable of inhibiting the proteolytic generation of $A \beta$ peptides from the APP holoprotein.

Formation of A $\beta$ from APP holoprotein is schematically depicted in Figure 1. Proteolytic processing of APP mediated via $\alpha$-secretase precludes $A \beta$ formation and leads to liberation of $\alpha$-sAPP, a soluble $\mathrm{NH}_{2}$-terminal ectodomain of APP that contains residues $1-15$ of the A $\beta$ peptide, which are believed to possess neuroprotective and/or neurotrophic properties. The secretion of $\alpha$-sAPP appears to be regulated through protein phosphoryla- tion via protein kinase C (22). Alternatively, APP processing through the $\beta$-, $\gamma$-secretase pathway (Figure 1) leads initially to formation of potentially amyloidogenic membrane-associated fragments and ultimately to the formation of intact $A \beta$ peptides. In vitro studies have suggested that $\beta$-secretase activity initiates the amyloidogenic catabolism of APP, suggesting that selective inhibitors of $\beta$-secretase could provide an attractive means to limit the formation of $A \beta$ in vivo. Conceivably, modulators of $\beta$-secretase could then funnel more APP holoprotein out of the amyloidogenic $A \beta$ pathway and into the $\alpha$-secretase pathway, thus generating higher levels of $\alpha$-sAPP at the expense of A $\beta$ peptides.

In our search for modulators of $\beta$-secretase, we set up a battery of immunoassays in order to quantify all of the major APP proteolytic products from the various cellular processing pathways that might be expressed in cell culture systems. In particular, we developed sandwich ELISA assays and/or immunoblot assays to measure total secreted APPs or sAPPs (i.e., $\alpha$-sAPP and $\beta$-sAPP), full-length holo-APPs, intracellular $\mathrm{COOH}$-terminal fragments derived from either $\beta$-secretase cleavage or $\alpha$-secretase cleavage (termed i $\beta$ and $i \alpha$, respectively), as well as the 2 major secreted $A \beta$ peptides $\left(A \beta_{40}\right.$ and $\left.A \beta_{42}\right)$. Using human glioblastoma cells transfected with various constructs harboring either wild-type $\left(\mathrm{APP}_{695}\right)$ or mutant $\mathrm{APP}_{695} \mathrm{cDNAs}$ ("Swedish" ${ }_{670,671}$ and/or "Indiana" ${ }_{717}$ ), we embarked on a lead compound discovery program. Our goal was to identify compounds capable of modulating the ratio of specific APP proteolytic fragments in such a way that they 
reduced or eliminated production of $A \beta$ peptides without affecting the level of APP holoprotein.

While evaluating a collection of commercially available protease inhibitors, we identified $N$-acetyl-L-leucine-Lleucine-L-norleucine aldehyde, calpain inhibitor I, as a reasonably effective modulator of APP amyloidogenic metabolism. Formation of $A \beta_{40}$ was significantly inhibited $\left(\mathrm{IC}_{50}=15 \mu \mathrm{M}\right)$, yet there was no observed effect on the levels of the APP holoprotein. These findings strongly suggested that calpain inhibitor I might attenuate the catabolism of APP, rather than exert generalized effects on the levels of protein expression. Interestingly, the structurally related compound $N$-acetyl-L-leucine-Lleucine-methionine aldehyde, calpain inhibitor II, was ineffective as an inhibitor of $A \beta_{40}$ formation. Thus, initial results suggested that inhibitors of the calpain inhibitor I class might constitute interesting lead compounds, and a structure-activity relationship (SAR) study was undertaken involving synthesis of di- and tripeptidyl aldehyde analogs having different amino acid side chains and distinct terminal functional groups.

The activity of calpain inhibitor I was dramatically improved during the course of the SAR study eventually affording a novel compound, SIB-1281, as the most active molecule in a series of structurally related peptidyl aldehydes. SIB-1281 is a tripeptidyl aldehyde containing a carboxylbenzyl capping group at the $\mathrm{NH}_{2}$-terminus and a 2methylpropene-containing unnatural amino acid side chain at $\mathrm{P}_{1}$. SIB-1281 exhibited the profile expected for a $\beta$-secretase inhibitor in the cell-based in vitro assays, decreasing both $A \beta_{40}$ and $A \beta_{42}\left(I_{50}=800 \mathrm{nM}\right)$ and concomitantly potentiating production of $\alpha$-sAPP $\left(\mathrm{EC}_{50}=\right.$ $650 \mathrm{nM}$ ). In addition, SIB-1281 had no effect on steadystate levels of APP holoprotein. Another analog from this series, SIB-1948, which retains the 2-methylpropene-containing unnatural amino acid side chain residue in $\mathrm{P}_{1}$, but substitutes a phenylalanine in $\mathrm{P}_{2}$, has nearly identical inhibitory properties to those of SIB-1281.

The inhibitory effects of SIB-1281 on cellular production of different $A \beta$ peptides were further evaluated using immunoprecipitation combined with matrixassisted laser desorption ionization time-of-flight (MALDI-TOF) mass. This approach enables a comprehensive analysis of all $\mathrm{A} \beta$ peptide variants and is the only type of mass spectrometry capable of identifying peptides or proteins. Human glioblastoma cells stably expressing an APP cDNA construct containing both the "Swedish" double mutation and the "Indiana" mutation were treated with either SIB-1281 or SIB-1405. Treatment of these cells with SIB-1281 $(10 \mu \mathrm{M})$ for 16 hours resulted in greater than $95 \%$ inhibition of $A \beta$ production (Figure 2), including the $A \beta$ peptide variants $A \beta_{37}, A \beta_{38}$, $A \beta_{40}$, and $A \beta_{42}$. In addition, SIB- 1281 elicited a substantial increase in $\alpha$-sAPP secretion ( 10 -fold).

Unlike SIB-1281, SIB-1405, a structurally related dipeptidyl aldehyde, inhibited formation of $\mathrm{A} \beta\left(\mathrm{IC}_{50}=3 \mu \mathrm{M}\right)$ but did not potentiate $\alpha$-sAPP secretion. Like the previous analogs in this series, SIB-1405 contains the 2-methylpropene unnatural amino acid side chain at $\mathrm{P}_{1}$; however, the $\mathrm{NH}_{2}$-terminus is capped with a 2-(S)-2-benzyoxy-4methylpentanoate. More surprising, according to the MALDI-TOF mass spectrometry, treatment with SIB-1405
$(10 \mu \mathrm{M})$ inhibited all detectable $A \beta$ variants but not $A \beta_{42}$. These data suggest that $A \beta_{40}$ and $A \beta_{42}$ may be processed by different $\gamma$-secretase activities, one of which is inhibitable by the dipeptidyl-aldehyde SIB-1405 and the tri-peptidyl aldehyde SIB-1281, and the other, by only SIB-1281 (Figure 2).

Interestingly, the profile exhibited by SIB-1405 is similar to that proposed for other " $\gamma$-secretase inhibitors" and is consistent with the biochemical expectations of $\gamma$-secretase inhibition. Because the $\gamma$-secretase cleavage appears not to be rate determining, inhibition of this event should inhibit $A \beta$ formation without a dramatic effect on the levels of $\alpha$-sAPP. Such $\gamma$-secretase modulators should also prevent the membrane-associated $\mathrm{COOH}$-terminal fragments (the initial products of $\alpha$ - and $\beta$-secretase cleavages, $i \alpha$ and i $\beta$, respectively) from being processed by $\gamma$-secretase. As a result, inhibitors of $\gamma$-secretase should transiently increase the concentration of $i \alpha$ and i $\beta$ intracellularly. The precise molecular targets of SIB-1281 and SIB-1405 are presently unknown, but both appear to exert effects on a proteolytic event within this amyloidogenic pathway. This conclusion is based both on their enzyme inhibitory profiles and their specific inhibitory effects on only certain APP proteolytic fragments.

While it is unlikely these peptidyl-aldehydes will ever become therapeutic candidates for $\mathrm{AD}$, due in part to their poor pharmacokinetic properties, they provide excellent tools with which to profile other putative $\beta$-secretase or $\gamma$-secretase modulators in cell-based assay sys-

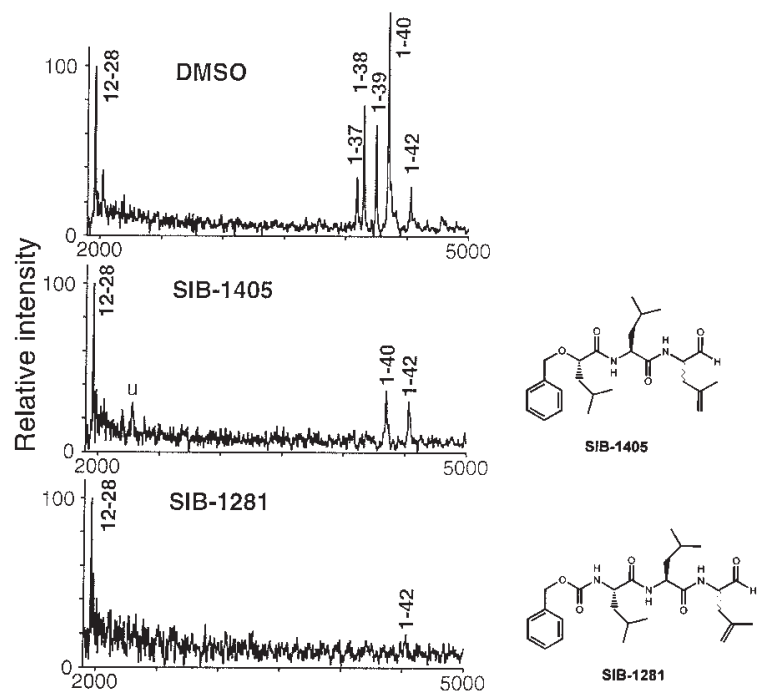

Figure 2

MALDI-TOF mass spectrometry of A $\beta$ peptide variants in conditioned medium of human glioblastoma cells stably transfected with an APP cDNA harboring a triplet of FAD-linked missense mutations ("Swedish" ${ }_{670 / 671}$ plus "Indiana" ${ }_{717}$ ). In the top panel, the cells were treated with the dimethyl sulfoxide $(0.1 \% \mathrm{vol} / \mathrm{vol})$ vehicle alone. The medium was harvested, immune precipitated with the $\mathrm{mAb} 4 \mathrm{G} 8$, and subjected to MALDI-TOF analysis. In the middle panel, the cells were treated with $10 \mu \mathrm{M}$ SIB-1405 in $0.1 \%$ DMSO for 16 hours. The medium was analyzed as described in the text. In the bottom panel, the cells were treated with $10 \mu \mathrm{M}$ SIB- 1281 in $0.1 \%$ DMSO for 16 hours, and medium was prepared as described in the text. The $12-28 \mathrm{~A} \beta$ peptide standard serves as a positive control that is added to medium before the immune precipitation step in order to assess quantitative recovery of peptides. 
tems. In addition, this series of compounds clearly demonstrates the value of cell-based immunochemical assay methods for identifying lead compounds suitable for SAR, preclinical development, and eventual advancement to the status of investigational new drugs for delaying the progression for $\mathrm{AD}$.

In vivo proof of principle. Several excellent transgenic mouse models exist today that permit studies of human APP proteolysis, $A \beta$ deposition, or neuritic plaque formation. To date, several groups have applied cDNA-based overexpression of mutant APPs and have successfully generated AD-type abnormalities in mouse brain (3). Another elegant and perhaps more genetically accurate approach has been to introduce entire genomic copies of either wild-type or mutant human APPs, as well as mutant human PS1 using yeast artificial chromosomes (YACs; ref. 23). The genomic APP-YAC constructs contain the transcriptional regulatory elements enabling correct temporal and spatial central nervous system expression and allow the entire spectrum of alternatively spliced transcripts and/or isoforms.

Regardless of whether a brain-selective cDNA-based overexpression system is used or entire genomic sequences are introduced with YACs, coexpression of mutant APP with mutant PS1 in transgenic mice leads to elevated levels of $A \beta_{42}$ and accelerated neuritic plaque formation. In our experience, the extent of amyloid deposition and the rate at which amyloid deposits and/or neuritic plaques develop in such transgenic models appears to rely primarily on levels of $A \beta_{42}$ expression. Indeed, when introduced into human APP-expressing transgenic mouse lines, human PS cDNAs encoding FAD-linked mutations are capable of increasing the level of $A \beta_{42}$ production, as well as accelerating the rate of amyloid deposition (23). These findings, which have been replicated by numerous laboratories around the world, strongly support the dependence of amyloid deposition and/or neuritic plaque formation on $A \beta_{42}$ production.

These transgenic models could serve as extremely useful reagents for assessing the in vivo efficacy of compounds, such as those described previously, that are capable of eliminating, or at least diminishing, $A \beta$ levels in cultured cell systems. In fact, compounds that have been shown to reduce $A \beta$ peptide levels in transgenic mice are expected to reach the clinic soon. These animal models can also be used to analyze the effects of pharmacologically reducing cerebral and/or vascular $A \beta$ peptide pools on the overall pathological phenotype. Although none of the currently available models recapitulate the entire scope of behavioral and pathological features of $\mathrm{AD}$, they do provide excellent tools for exam- ining not only potential drugs, but also genetic and environmental modifiers of this dreaded disease.

\section{Acknowledgments}

The authors acknowledge G. Holtz, P. Baskin, and B. Rowe for technical support, and R. Wang of The Rockefeller University for conducting the MALDI-TOF analysis.

1. Goate, A., et al. 1991. Segregation of a missense mutation in the amyloid precursor protein gene with familial Alzheimer's disease. Nature. 349:704-706.

2. Sherrington, R., et al. 1995. Cloning of a gene bearing missense mutations in early-onset familial Alzheimer's disease. Nature. 375:754-760.

3. Price, D.L., and Sisodia, S.S. 1998. Mutant genes in familial Alzheimer's disease and transgenic models. Annu. Rev. Neurosci. 21:479-505.

4. Levy-Lahad, E., et al. 1995. Candidate gene for the chromosome 1 familial Alzheimer's disease locus. Science. 269:973-977.

5. Shoji, M., et al. 1992. Production of the Alzheimer amyloid $\beta$ protein by normal proteolytic processing. Science. 258:126-129.

6. Hardy, J. 1992. Framing $\beta$-amyloid. Nat. Genet. 1:233-234.

7. Cai, X.D., Golde, T.E., and Younkin, S.G. 1993. Release of excess amyloid $\beta$ protein from a mutant amyloid $\beta$ protein precursor. Science. 259:514-516.

8. Iwatsubo, T., et al. 1994. Visualization of $A \beta 42(43)$ and $A \beta 40$ in senile plaques with end-specific $A \beta$ monoclonals: evidence that an initially deposited species is A $342(43)$. Neuron. 13:45-53.

9. Jarrett, J.T., and Lansbury, P.T., Jr. 1993. Seeding "one-dimensional crystallization" of amyloid: a pathogenic mechanism in Alzheimer's disease and scrapie? Cell. 73:1055-1058.

10. Scheuner, D., et al. 1996. Secreted amyloid $\beta$-protein similar to that in the senile plaques of Alzheimer's disease is increased in vivo by the presenilin 1 and 2 and APP mutations linked to familial Alzheimer's disease. Nat. Med. 8:864-870.

11. Loo, D.T., et al. 1993. Apoptosis is induced by $\beta$-amyloid in cultured central nervous system neurons. Proc. Natl. Acad. Sci. USA. 90:7951-7955.

12. Behl, C., Davis, J.B., Lesley, R., and Schubert, D. 1994. Hydrogen peroxide mediates amyloid $\beta$ protein toxicity. Cell. 77:817-827.

13. Akama, K.T., Albanese, C., Pestell, R.G., and Van Eldik, L.J. 1998. Amyloid $\beta$-peptide stimulates nitric oxide production in astrocytes through an NFKB-dependent mechanism. Proc. Natl. Acad. Sci. USA. 95:5795-5800.

14. Hensley, K., et al. 1994. A model for $\beta$-amyloid aggregation and neurotoxicity based on free radical generation by the peptide: relevance to Alzheimer's disease. Proc. Natl. Acad. Sci. USA. 91:3270-3274.

15. Rogers, J., et al. 1992. Complement activation by $\beta$-amyloid in Alzheimer's disease. Proc. Natl. Acad. Sci. USA. 89:10016-10020.

16. Kingston, I.B., Castro, M.J., and Anderson, S. 1995. In vitro stimulation of tissue-type plasminogen activator by Alzheimer amyloid $\beta$-peptide analogues. Nat. Med. 2:138-142.

17. Boggs, L.N., et al. 1996. Clusterin (Apo J) protects against in vitro amyloid$\beta(1-40)$ neurotoxicity. J. Neurochem. 67:1324-1327.

18. Chen, S., Frederickson, C.A., and Brunden, K.R. 1996. Neuroglial-mediated immunoinflammatory responses in Alzheimer's disease: complement activation and therapeutic approaches. Neurobiol. Aging. 17:781-787.

19. Tjernberg, L.O., et al. 1997. Controlling amyloid $\beta$-peptide fibril formation with protease-stable ligands. J. Biol. Chem. 272:12601-12605.

20. Solomon, B., Koppel, R., Frankel, D., and Hanan-Aharon, E. 1997. Disaggregation of Alzheimer $\beta$-amyloid by site-directed mAb. Proc. Natl. Acad. Sci. USA. 94:4109-4112.

21. Esler, W.P., et al. 1997. A beta deposition inhibitor screen using synthetic amyloid. Nat. Biotechnol. 15:258-263.

22. Caporaso, G.L., Gandy, S.E., Buxbaum, J.D., Ramabhadran, T.V., and Greengard, P. 1992. Protein phosphorylation regulates secretion of Alzheimer ß/A4 amyloid precursor protein. Proc. Natl. Acad. Sci. USA. 89:3055-3059.

23. Lamb, B.T., et al. 1999. Amyloid production and deposition in mutant amyloid precursor protein and presenilin-1 yeast artificial chromosome transgenic mice. Nat. Neurosci. 2:695-697. 